\title{
Study of elementary deformation mechanisms in MgO induced by nanoindentation: experiments and simulation
}

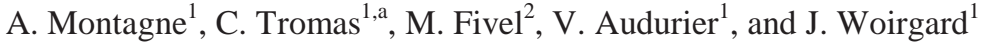 \\ ${ }^{1}$ Université de Poitiers, Laboratoire PhyMat, 86962 Futuroscope Chasseneuil, France \\ ${ }^{2}$ CNRS Grenoble, Laboratoire SIMaP groupe GPM2, 38402 Saint Martin d'Hères Cedex, France
}

Nanoindentation test is a common method to study mechanical properties at a nanometre scale such as hardness or Young's modulus [1]. This mechanical test consists in applying a force on the sample through a diamond tip while the resulting penetration depth is monitored during a loading-unloading cycle. Mechanical properties can be extracted from the so called load-displacement curve thanks to analysis model. The nanoindentation test can be also used to induce locally plastic deformation and thus to study incipient plasticity. In particular, as very high stress can be reached in material volumes small enough to be considered as dislocations free, this method is interesting to investigate the dislocation nucleation process. In the present study this method has been applied in single crystals of Magnesium Oxide $(\mathrm{MgO})$ which have been chosen as a model material due to its rather simple plasticity.

A spherical indenter with a high radius $(10 \mu \mathrm{m})$ has been used for two reasons. Firstly, the elastic contact between a sphere and a flat surface is well described by the Hertz law [2]. And secondly, the stress induced by a spherical contact is easily calculable.

Residual deformations around nanoindentation imprint have been observed by Atomic Force Microscope (AFM). The high resolution of the AFM and its ability to measure height variations as low as few Angstroms is of particular interest to analyse slip lines due to the emergence and propagation of dislocations around the indents. AFM has also been associated to a nanoetching technique which consists in revealing the emergence point of dislocations, combined with a fine chemomechanical polishing to perform a $3 \mathrm{D}$ reconstruction of the dislocation structure [3].

Classically, plastic deformation and imprint formation during a nanoindentation test are related to an hysteresis cycle in the load displacement curve. However, in few cases, AFM observation of indented areas revealed small residual deformation, whereas the corresponding load-displacement curves exhibited a fully reversible behaviour. Furthermore, the fine analysis of these experimental loading curves showed a deviation from the Hertz law for penetration depth higher than 100nm. From the analysis of the stress field in particular slip planes, the hypothesis of reversible plasticity has been made.

In order to validate the hypothesis made from the experimental observations, experimental tests have been modelled using a combination of three-dimensional discrete dislocation simulation (DDD) and the finite element method (FEM) [4]. A virtual sample has been build with finite elements, and the experimental indentation parameters have been used in the FEM code. The contact induced stress field calculated from the FEM is the used in the DDD simulation to calculate the equilibrium position of dislocations below the indenter.

\footnotetext{
a e-mail: christophe.tromas@univ-poitiers.fr
}

This is an Open Access article distributed under the terms of the Creative Commons Attribution-Noncommercial License 3.0, which permits unrestricted use, distribution, and reproduction in any noncommercial medium, provided the original work is properly cited. 
Dislocation loops are progressively introduced at the point of maximum resolved shear stress in the identified experimental slip plane. Their equilibrium shape and position are then calculated under load and during the indenter unloading. When less than 30 dislocation loops are introduced, they form a pile up in the volume without emerging at the surface. When the load is progressively removed, the decreasing stress induced by the indenter drains back the dislocations until their line tension as well as the pile up back stress lead them to annihilate.

1. W.C. Oliver, and G.M. Pharr, Journal of Materials Research 7, 1564 (1992)

2. B.R. Lawn, Journal of the American Ceramic Society 81, 1977 (1998)

3. Y. Gaillard, C. Tromas, and J. Woirgard, Acta Materialia 51, 1059 (2003)

4. M.C. Fivel, C.F. Robertson, G.R. Canova, and L. Boulanger, Acta Materialia 46, 6183 (1998) 